\title{
An Elliptic Neumann Problem with Subcritical Nonlinearity
}

by

\author{
Jan CHABROWSKI and Kyril TINTAREV
}

Presented by Bogdan BOJARSKI

Summary. We establish the existence of a solution to the Neumann problem in the halfspace with a subcritical nonlinearity on the boundary. Solutions are obtained through the constrained minimization or minimax. The existence of solutions depends on the shape of a boundary coefficient.

1. Introduction. Let $\mathbb{R}_{+}^{N}=\mathbb{R}^{N-1} \times(0, \infty)$. For a point $x \in \mathbb{R}_{+}^{N}=$ $\mathbb{R}^{N-1} \times(0, \infty)$ we use the notation $x=\left(x^{\prime}, x_{N}\right)$, where $x^{\prime} \in \mathbb{R}^{N-1}$ and $x_{N}$ $>0$. In this paper we consider a semilinear Neumann problem in $H^{1}\left(\mathbb{R}_{+}^{N}\right)$, $N>2$,

$$
\left\{\begin{array}{l}
-\Delta u+u=0 \quad \text { in } \mathbb{R}_{+}^{N}, \\
\frac{\partial u\left(x^{\prime}, 0\right)}{\partial x_{N}}=b\left(x^{\prime}\right) u^{p-1}\left(x^{\prime}, 0\right) \quad \text { on } \mathbb{R}^{N-1}, \quad u>0 \quad \text { on } \mathbb{R}_{+}^{N},
\end{array}\right.
$$

where $p \in(2,2(N-1) /(N-2))$ and $b \in L^{\infty}\left(\mathbb{R}^{N-1}\right)$. It is well known that the trace embedding of the Sobolev space $H^{1}\left(\mathbb{R}_{+}^{N}\right)$ into $L^{p}\left(\mathbb{R}^{N-1}\right)$, $p \in(2,2(N-1) /(N-2))$ is continuous but not compact. The norm in $H^{1}\left(\mathbb{R}_{+}^{N}\right)$ is defined by

$$
\|u\|^{2}=\int_{\mathbb{R}_{+}^{N}}\left(|\nabla u|^{2}+u^{2}\right) d x .
$$

It is assumed that $\lim _{\left|x^{\prime}\right| \rightarrow \infty} b\left(x^{\prime}\right)=b_{\infty}>0$.

In this paper we prove existence when (i) $b\left(x^{\prime}\right)>b_{\infty}$ on $\mathbb{R}^{N-1}$ or (ii) $b\left(x^{\prime}\right)$ $>m^{-(p-2) / 2} b_{\infty}$ on $\mathbb{R}^{N-1}$, provided that $b$ is invariant with respect to a finite

2000 Mathematics Subject Classification: 35B33, 35B38, 35J35, 35J60, 35J65, 35Q55.

Key words and phrases: trace embedding, concentration-compactness principle, subcritical exponent. 
subgroup of $O\left(\mathbb{R}^{N-1}\right)$ of cardinality $m$ acting freely on $\mathbb{R}^{N-1} \backslash\{0\}$. We also consider the case when the above penalty condition is reversed: $b\left(x^{\prime}\right)<b_{\infty}$ on $\mathbb{R}^{N-1}$. However, in this case we only present a partial result (see Theorem 1.4) which depends on the convexity of $b\left(x^{\prime}\right)$.

The main results of this paper are the following:

THEOREM 1.1. Suppose that $b\left(x^{\prime}\right)$ is a $\mathbb{Z}^{N-1}$-periodic function. Then problem (1.1) admits a solution.

TheOREM 1.2. Suppose that $b \in L^{\infty}\left(\mathbb{R}^{N-1}\right)$ and that $b_{\infty}<b\left(x^{\prime}\right)$ on $\mathbb{R}^{N-1}$. Then problem (1.1) admits a solution.

THEOREM 1.3. Suppose that $b\left(x^{\prime}\right)$ is invariant with respect to a finite subgroup $G \subset O\left(\mathbb{R}^{N-1}\right)$ of cardinality $m$ acting freely on $\mathbb{R}^{N-1} \backslash\{0\}$ and that

$$
b\left(x^{\prime}\right)>m^{-(p-2) / 2} b_{\infty} \quad \text { for } x^{\prime} \in \mathbb{R}^{N-1} .
$$

Then problem (1.1) admits a $G$-invariant solution.

The proofs of Theorems 1.1 and 1.2 are standard. Solutions are obtained as multiples of minimizers of the constrained minimization problem

$$
c_{b}=\inf _{u \in H^{1}\left(\mathbb{R}_{+}^{N}\right), \int_{\mathbb{R}^{N-1}} b\left(x^{\prime}\right)\left|u\left(x^{\prime}, 0\right)\right|^{p} d x^{\prime}=1} \int_{\mathbb{R}_{+}^{N}}\left(|\nabla u|^{2}+u^{2}\right) d x .
$$

In the case of the proof of Theorem 1.3 the space $H^{1}\left(\mathbb{R}_{+}^{N}\right)$ in the above minimization problem will be replaced by a subspace of $G$-invariant functions in $x^{\prime}$. Similar results are known for the equation

$$
-\Delta u+u=|u|^{p-2} u \quad \text { on } \mathbb{R}^{N},
$$

where $1<p<2 N /(N-2)$ (see [4], [6]).

TheOrem 1.4. Assume that $b \in L^{\infty}\left(\mathbb{R}^{N-1}\right)$ is such that

$$
b\left(x^{\prime}\right)<b_{\infty} \quad \text { for } x \in \mathbb{R}^{N-1} .
$$

Then there exists a finite set $Y \subset \mathbb{Z}^{N-1}$ and $c_{y^{\prime}} \in[0,1], y^{\prime} \in Y, \sum_{Y} c_{y^{\prime}}=1$, such that problem (1.1) with $b^{Y}\left(x^{\prime}\right)=\sum_{Y} c_{y^{\prime}} b\left(x^{\prime}-y^{\prime}\right)$ in place of $b\left(x^{\prime}\right)$ has a solution.

Note that $b^{Y}\left(x^{\prime}\right)<b_{\infty}^{Y}=b_{\infty}$. We do not know if existence holds for every $b$, or whether convexity is essential for the existence. If $b$ is radially symmetric, problem (1.1) admits a solution radially symmetric in the variables $x^{\prime}$ obtained as a multiple of a minimizer of the problem

$$
\inf _{\int_{\mathbb{R}^{N-1}} b\left(x^{\prime}\right)\left|u\left(x^{\prime}, 0\right)\right|^{p} d x^{\prime}=1, u \in H_{\mathrm{r}}^{1}\left(\mathbb{R}_{+}^{N}\right)} \int_{\mathbb{R}_{+}^{N}}\left(|\nabla u|^{2}+u^{2}\right) d x,
$$

where $H_{\mathrm{r}}^{1}\left(\mathbb{R}_{+}^{N}\right)$ is a subspace of $H^{1}\left(\mathbb{R}_{+}^{N}\right)$ consisting of functions radially symmetric in $x^{\prime}$. The existence of a minimizer follows from the compactness 
of the trace embedding of $H_{\mathrm{r}}^{1}\left(\mathbb{R}_{+}^{N}\right)$ into a subspace of radially symmetric functions in $L^{p}\left(\mathbb{R}^{N-1}\right), p \in\left(2,2^{2(N-1) /(N-2)}\right)$.

2. Global compactness. Theorem 2.2 below is a particular case of the functional-analytic global compactness theorem from [5], applied to the Sobolev space $H^{1}\left(\mathbb{R}_{+}^{N}\right), N>2$, with the norm $\|\cdot\|$ and the dislocations defined by shifts $u \mapsto u\left(\cdot-y^{\prime}, \cdot\right), y^{\prime} \in \mathbb{Z}^{N-1}$. The derivation of this particular case is completely analogous to the case of $H^{1}\left(\mathbb{R}^{N}\right)$ with shifts by $y \in \mathbb{Z}^{N}$ elaborated in [5], once one takes into account the following statement, close to the one from [3], which deals with convergence in $L^{p}\left(\mathbb{R}^{N}\right)$.

LEMMA 2.1. Let $u_{k}$ be a bounded sequence in $H^{1}\left(\mathbb{R}_{+}^{N}\right)$ and let $p \in$ $(2,2(N-1) /(N-2))$. Then $u_{k}\left(\cdot+y_{k}^{\prime}, \cdot\right) \rightarrow 0$ for all $y_{k}^{\prime} \in \mathbb{Z}^{N-1}$ implies $\left\|u_{k}\right\|_{L^{p}\left(\mathbb{R}^{N-1}\right)} \rightarrow 0$.

Proof. Assume that $u_{k}\left(\cdot+y_{k}^{\prime}, \cdot\right) \rightarrow 0$ for any $y_{k}^{\prime} \in \mathbb{Z}^{N-1}$. Consider a unit cube $Q:=(0,1)^{N-1}$. By the trace inequality for bounded domains, there is a $C>0$ such that

$$
\begin{aligned}
& \int_{Q+y^{\prime}}\left|u_{k}\left(x^{\prime}, 0\right)\right|^{p} d x^{\prime} \\
& \leq C\left\|u_{k}\right\|_{H^{1}\left(\left(Q+y^{\prime}\right) \times(0, \infty)\right)}^{2}\left(\int_{Q+y^{\prime}}\left|u_{k}\left(x^{\prime}, 0\right)\right|^{p} d x^{\prime}\right)^{1-2 / p}
\end{aligned}
$$

for all $y^{\prime} \in \mathbb{Z}^{N-1}$. By adding (2.1) over $y^{\prime} \in \mathbb{Z}^{N-1}$, and noting that the union $\bigcup_{y^{\prime} \in \mathbb{Z}^{N-1}}\left(Q+y^{\prime}\right)$ is $\mathbb{R}^{N-1}$ up to a set of measure zero, we obtain

$$
\begin{aligned}
\int_{\mathbb{R}^{N-1}}\left|u_{k}\left(x^{\prime}, 0\right)\right|^{p} d x^{\prime} & \leq C\left\|u_{k}\right\|^{2} \sup _{y^{\prime} \in \mathbb{Z}^{N-1}}\left(\int_{Q}\left|u_{k}\left(x^{\prime}+y^{\prime}, 0\right)\right|^{p} d x^{\prime}\right)^{1-2 / p} \\
& \leq 2 C\left\|u_{k}\right\|^{2}\left(\int_{Q}\left|u_{k}\left(x^{\prime}+y_{k}^{\prime}, 0\right)\right|^{p} d x^{\prime}\right)^{1-2 / p}
\end{aligned}
$$

where $y_{k}^{\prime} \in \mathbb{Z}^{N-1}$ is any sequence satisfying

$$
\begin{aligned}
\left(\int_{Q}\left|u_{k}\left(x^{\prime}+y_{k}^{\prime}, 0\right)\right|^{p} d x^{\prime}\right)^{1-2 / p} & \\
& \geq \frac{1}{2} \sup _{y^{\prime} \in \mathbb{Z}^{N-1}}\left(\int_{Q}\left|u_{k}\left(x^{\prime}+y^{\prime}, 0\right)\right|^{p} d x^{\prime}\right)^{1-2 / p} .
\end{aligned}
$$

It remains to note that by compactness of the trace of $H^{1}(Q \times(0, \infty))$ into $L^{p}(Q)$, one has $u_{k}\left(\cdot+y_{k}^{\prime}, 0\right) \rightarrow 0$ in $L^{p}\left(\mathbb{R}^{N-1}\right)$, so that the assertion of the lemma follows from $(2.2)$.

ThEOREM 2.2. Let $\left\{u_{k}\right\} \subset H^{1}\left(\mathbb{R}_{+}^{N}\right)$ be a bounded sequence. Then there exist $w^{(n)} \in H^{1}\left(\mathbb{R}_{+}^{N}\right), y_{k}^{(n)^{\prime}} \in \mathbb{Z}^{N-1}, y_{k}^{(1)^{\prime}}=0$, with $k, n \in \mathbb{N}$, such that for $a$ 
relabelled subsequence,

$$
\begin{gathered}
w^{(n)}=\underset{k \rightarrow \infty}{w-\lim } u_{k}\left(\cdot+y_{k}^{(n)^{\prime}}, \cdot\right) \\
\left|y_{k}^{(n)^{\prime}}-y_{k}^{(m)^{\prime}}\right| \rightarrow 0 \quad \text { as } k \rightarrow \infty \text { for } n \neq m, \\
\sum_{n \in \mathbb{N}}\left\|w^{(n)}\right\|^{2} \leq \limsup \left\|u_{k}\right\|^{2}, \\
u_{k}-\sum_{n \in \mathbb{N}} w^{(n)}\left(\cdot-y_{k}^{(n)^{\prime}}, \cdot\right) \rightarrow 0 \quad \text { in } L^{p}\left(\mathbb{R}^{N-1}\right) \\
\text { as } k \rightarrow \infty, p \in\left(2, \frac{2(N-1)}{N-2}\right),
\end{gathered}
$$

where the series $\sum_{n \in \mathbb{N}} w^{(n)}\left(\cdot-y_{k}^{(n)^{\prime}}, \cdot\right)$ converges uniformly in $k$.

The following lemma is a variant of the Brézis-Lieb lemma from [1].

Lemma 2.3. Let $b \in L^{\infty}\left(\mathbb{R}^{N-1}\right)$ and assume that $b\left(x^{\prime}\right) \rightarrow b_{\infty} \in \mathbb{R}$ as $\left|x^{\prime}\right| \rightarrow \infty$. Let $u_{k}, w^{(n)}$, and $y_{k}^{(n)^{\prime}}$ be as in Theorem 2.2. Then for every $p \in(2,2(N-1) /(N-2)), y^{\prime} \in \mathbb{Z}^{N-1}$,

$$
\begin{aligned}
\lim _{k \rightarrow \infty} & \int_{\mathbb{R}^{N-1}} b\left(x^{\prime}\right)\left|u_{k}\left(x^{\prime}+y^{\prime}, 0\right)\right|^{p} d x^{\prime} \\
\quad & =\int_{\mathbb{R}^{N-1}} b\left(x^{\prime}\right)\left|w^{(1)}\left(x^{\prime}+y^{\prime}, 0\right)\right|^{p} d x^{\prime}+\sum_{n \geq 2} \int_{\mathbb{R}^{N-1}} b_{\infty}\left|w^{(n)}\left(x^{\prime}, 0\right)\right|^{p} d x^{\prime}
\end{aligned}
$$

and the convergence is uniform in $y^{\prime}$.

Proof. First we note that the statement easily reduces to the case $y=0$ due to the convergence of $b\left(x^{\prime}\right)$ to $b_{\infty}$ as $\left|x^{\prime}\right| \rightarrow \infty$, once one considers the left hand side of $(2.7)$ as $\lim _{k \rightarrow \infty} \int_{\mathbb{R}^{N-1}} b\left(x^{\prime}-y^{\prime}\right)\left|u_{k}\left(x^{\prime}, 0\right)\right|^{p} d x^{\prime}$. For the case $y=0$ we give a sketch of the proof only, since similar statements have been proved several times elsewhere. In view of Lemma 2.1 we may assume that $u_{k}=\sum_{n \in \mathbb{N}} w^{(n)}\left(\cdot-y_{k}^{(n)^{\prime}}, \cdot\right)$. Since the series is absolutely convergent and $u \mapsto \int_{\mathbb{R}^{N-1}} b\left(x^{\prime}\right)\left|u\left(x^{\prime}, 0\right)\right|^{p} d x^{\prime}$ is continuous in $H^{1}\left(\mathbb{R}_{+}^{N}\right)$, it suffices to prove the lemma if the sum has finitely many terms. By density of $\left.C_{0}^{\infty}\left(\mathbb{R}^{N}\right)\right|_{\mathbb{R}_{+}^{N}}$ in $H^{1}\left(\mathbb{R}_{+}^{N}\right)$, it suffices to prove the lemma when $\left.w^{(n)} \in C_{0}^{\infty}\left(\mathbb{R}^{N}\right)\right|_{\mathbb{R}_{+}^{N}}$. Since $\left|y_{k}^{(n)^{\prime}}-y_{k}^{(m)^{\prime}}\right| \rightarrow \infty$ for $m \neq n$, there is a $k_{0}$ such that for all $k \geq k_{0}$ all the functions $w^{(n)}\left(\cdot-y_{k}^{(n)^{\prime}}, \cdot\right)$ have disjoint supports. In this case

$$
\begin{aligned}
\int_{\mathbb{R}^{N-1}} b\left(x^{\prime}\right)\left|u_{k}\left(x^{\prime}, 0\right)\right|^{p} d x^{\prime}=\sum_{n \geq 1} \int_{\mathbb{R}^{N-1}} b\left(x^{\prime}+y_{k}^{(n)^{\prime}}\right)\left|w^{(n)}\left(x^{\prime}, 0\right)\right|^{p} d x^{\prime} \\
\rightarrow \int_{\mathbb{R}^{N-1}} b\left(x^{\prime}\right)\left|w^{(1)}\left(x^{\prime}, 0\right)\right|^{p} d x^{\prime}+\sum_{n \geq 2} \int_{\mathbb{R}^{N-1}} b_{\infty}\left|w^{(n)}\left(x^{\prime}, 0\right)\right|^{p} d x^{\prime} .
\end{aligned}
$$


3. Proofs of Theorems 1.1-1.3. The results of Section 2 will now be applied to prove Theorems 1.1-1.3.

Proof of Theorem 1.1. Let $\left\{u_{k}\right\} \subset H^{1}\left(\mathbb{R}_{+}^{N}\right)$ be a minimizing sequence for the constant $c_{b}$ with $\int_{\mathbb{R}^{N-1}} b\left(x^{\prime}\right)\left|u_{k}\left(x^{\prime}, 0\right)\right|^{p} d x^{\prime}=1$ for each $k$. We apply Theorem 2.2 with dislocations $g_{y_{k}^{\prime}}: u \mapsto u\left(\cdot+y_{k}^{\prime}, \cdot\right), y_{k}^{\prime} \in \mathbb{Z}^{N-1}$. Let $\left\{u_{k}\right\}$, $\left\{w^{(n)}\right\}$ and $\left\{y_{k}^{\prime(n)}\right\}$ be subsequences generated by Theorem 2.2. According to Theorem 2.2 , since $b\left(x^{\prime}\right)$ is periodic, we have

$$
1=\int_{\mathbb{R}^{N-1}} b\left(x^{\prime}\right)\left|u_{k}\left(x^{\prime}, 0\right)\right|^{p} d x^{\prime}=\sum_{n} \int_{\mathbb{R}^{N-1}} b\left(x^{\prime}\right)\left|w^{(n)}\left(x^{\prime}, 0\right)\right|^{p} d x^{\prime} .
$$

It follows from (2.5) that

$$
\sum_{n}\left\|w^{(n)}\right\|^{2} \leq c_{b}
$$

We now set $\int_{\mathbb{R}^{N-1}}\left|w^{(n)}\left(x^{\prime}, 0\right)\right|^{p} d x^{\prime}=t_{n}$. Obviously we have $\left\|t_{n}^{-1 / p} w^{(n)}\right\| \geq$ $c_{b}$, which yields $\left\|w^{(n)}\right\|^{2} \geq c_{b} t_{n}^{2 / p}$. Applying this to (3.2), we get

$$
\sum_{n} t_{n}^{2 / p} \leq 1
$$

On the other hand, we deduce from (3.1) that $\sum_{n} t_{n}=1$. Since $2 / p<1$, the last relation and $(3.3)$ can only hold if exactly one term $t_{n}$, say $t_{n_{\circ}}$, is nonzero and $t_{n}=0$ for all $n \neq n_{\circ}$. This yields $\left\|w^{\left(n_{\circ}\right)}\right\|^{2}=c_{b}$ and hence $w^{\left(n_{\circ}\right)}$ is a minimizer.

Corollary 3.1. Let $b\left(x^{\prime}\right)=1$ on $\mathbb{R}^{N-1}$. Then there exists a minimizer for $c_{b}$.

We now consider the case $b\left(x^{\prime}\right)>b_{\infty}$ on $\mathbb{R}^{N-1}$.

Proof of Theorem 1.2. Let $c_{\infty}=c_{b}$ with $b\left(x^{\prime}\right) \equiv b_{\infty}$. By Corollary 3.1 the constant $c_{\infty}$ is attained on a positive function $v$. Hence

$$
\begin{aligned}
c_{b} & \leq \frac{\int_{\mathbb{R}_{+}^{N}}\left(|\nabla v|^{2}+v^{2}\right) d x}{\left(\int_{\mathbb{R}^{N-1}} b\left(x^{\prime}\right)\left|v\left(x^{\prime}, 0\right)\right|^{p} d x^{\prime}\right)^{2 / p}} \\
& <\frac{\int_{\mathbb{R}_{+}^{N}}\left(|\nabla v|^{2}+v^{2}\right) d x}{\left(\int_{\mathbb{R}^{N-1}} b_{\infty}\left|v\left(x^{\prime}, 0\right)\right|^{p} d x^{\prime}\right)^{2 / p}}=c_{\infty} .
\end{aligned}
$$

Let $\left\{u_{k}\right\}$ be a minimizing sequence for $c_{b}$. We may assume that $u_{k} \rightarrow w$ in $H^{1}\left(\mathbb{R}_{+}^{N}\right)$ and also $u_{k} \rightarrow w$ in $L^{p}\left(\mathbb{R}^{N-1}\right)$. Setting

$$
a(u)=\int_{\mathbb{R}_{+}^{N}}\left(|\nabla u|^{2}+u^{2}\right) d x \quad \text { and } \quad v_{k}=u_{k}-w
$$

we can write

$$
c_{b}=a(w)+a\left(v_{k}\right)+o(1)
$$


up to a subsequence and by the Brézis-Lieb lemma [1] we also have

$$
\begin{aligned}
1=\int_{\mathbb{R}^{N-1}} b\left(x^{\prime}\right)\left|u_{k}\left(x^{\prime}, 0\right)\right|^{p} d x^{\prime}= & \int_{\mathbb{R}^{N-1}} b\left(x^{\prime}\right)\left|v_{k}\left(x^{\prime}, 0\right)\right|^{p} d x^{\prime} \\
& +\int_{\mathbb{R}^{N-1}} b\left(x^{\prime}\right)\left|w\left(x^{\prime}, 0\right)\right|^{p} d x^{\prime}+o(1) .
\end{aligned}
$$

We deduce from the last two relations that

$$
\begin{aligned}
c_{b} \geq & c_{b}\left(\int_{\mathbb{R}^{N-1}} b\left(x^{\prime}\right)\left|w\left(x^{\prime}, 0\right)\right|^{p} d x^{\prime}\right)^{2 / p} \\
& +c_{b}\left(\int_{\mathbb{R}^{N-1}} b\left(x^{\prime}\right)\left|v_{k}\left(x^{\prime}, 0\right)\right|^{p} d x^{\prime}\right)^{2 / p}+o(1) \\
= & c_{b}\left(\int_{\mathbb{R}^{N-1}} b\left(x^{\prime}\right)\left|w\left(x^{\prime}, 0\right)\right|^{p} d x^{\prime}\right)^{2 / p} \\
& +\left(1-\int_{\mathbb{R}^{N-1}} b\left(x^{\prime}\right)\left|w\left(x^{\prime}, 0\right)\right|^{p} d x^{\prime}\right)^{2 / p}+o(1) .
\end{aligned}
$$

We therefore have either

(i) $\int_{\mathbb{R}^{N-1}} b\left(x^{\prime}\right)\left|w\left(x^{\prime}, 0\right)\right|^{p} d x^{\prime}=1$ or

(ii) $\int_{\mathbb{R}^{N-1}} b\left(x^{\prime}\right)\left|w\left(x^{\prime}, 0\right)\right|^{p} d x^{\prime}=0$.

We show that (ii) cannot occur. Indeed, if $\int_{\mathbb{R}^{N-1}} b\left(x^{\prime}\right)\left|w\left(x^{\prime}, 0\right)\right|^{p} d x^{\prime}=0$, then $u_{k} \rightarrow 0$ in $H^{1}\left(\mathbb{R}_{+}^{N}\right)$ and in $L^{p}\left(\mathbb{R}^{N-1}\right)$ (in the sense of traces). Since $b\left(x^{\prime}\right) \rightarrow b_{\infty}$ as $\left|x^{\prime}\right| \rightarrow \infty$, we get

$$
1=\int_{\mathbb{R}^{N-1}} b\left(x^{\prime}\right)\left|u_{k}\left(x^{\prime}, 0\right)\right|^{p} d x^{\prime}=\int_{\mathbb{R}^{N-1}} b_{\infty}\left|u_{k}\left(x^{\prime}, 0\right)\right|^{p} d x^{\prime}+o(1) .
$$

This yields $c_{\infty} \leq c_{b}$, which contradicts (3.4). Hence case (i) holds and $w$ is a minimizer for $c_{b}$.

To prove Theorem 1.3 , we introduce the subspace $H_{G}^{1}\left(\mathbb{R}_{+}^{N}\right)$ of $H^{1}\left(\mathbb{R}_{+}^{N}\right)$ defined by

$$
H_{G}^{1}\left(\mathbb{R}_{+}^{N}\right)=\left\{u \in H^{1}\left(\mathbb{R}_{+}^{N}\right): u \circ \gamma=u \text { for all } \gamma \in G\right\}
$$

and set

$$
c_{b, G}=\sup _{\|u\|=1, u \in H_{G}^{1}\left(\mathbb{R}_{+}^{N}\right)} \int_{\mathbb{R}^{N-1}} b\left(x^{\prime}\right)\left|u\left(x^{\prime}, 0\right)\right|^{p} d x^{\prime} .
$$

We also need

$$
c_{\infty, G}=\sup _{\|u\|=1, u \in H_{G}^{1}\left(\mathbb{R}_{+}^{N}\right)} \int_{\mathbb{R}^{N-1}} b_{\infty}\left|u\left(x^{\prime}, 0\right)\right|^{p} d x^{\prime} .
$$


Observe that

$$
c_{\infty, G}=c_{\infty}:=\sup _{\|u\|=1, u \in H^{1}\left(\mathbb{R}_{+}^{N}\right)} \int_{\mathbb{R}^{N-1}} b_{\infty}\left|u\left(x^{\prime}, 0\right)\right|^{p} d x^{\prime} .
$$

Indeed, $c_{\infty, G} \leq c_{\infty}$ since $H_{G}^{1}\left(\mathbb{R}_{+}^{N}\right) \subset H^{1}\left(\mathbb{R}_{+}^{N}\right)$. Moreover, the standard argument based on spherical decreasing rearrangements (with respect to the $\mathbb{R}^{N-1}$-variable) implies that $c_{\infty}$ is attained on a radially symmetric function, that is, on $H_{G}^{1}\left(\mathbb{R}_{+}^{N}\right)$, and (3.5) is immediate. It then follows from (1.2) and (3.5) that

$$
c_{\infty}<m^{(p-2) / 2} c_{b, G}
$$

Proof of Theorem 1.3. Let $\left\{u_{k}\right\} \subset H_{G}^{1}\left(\mathbb{R}_{+}^{N}\right)$ be a maximizing sequence for the constant $c_{b, G}$. We apply Theorem 2.2 to the sequence $\left\{u_{k} \circ \gamma\right\}, \gamma \in G$. We have, by the $G$-invariance,

$$
\underset{k \rightarrow \infty}{w-\lim } u_{k}\left(\cdot+\gamma y_{k}^{(n)^{\prime}}, \cdot\right)=\underset{k \rightarrow \infty}{w-\lim } u_{k}\left(\gamma^{-1} \cdot+y_{k}^{(n)^{\prime}}, \cdot\right)=w^{(n)} \circ \gamma^{-1} .
$$

Let $n>1$. Since $G$ is a finite group whose nontrivial elements have no fixed points, $\left|\gamma y_{k}^{(n)^{\prime}}-\gamma^{\prime} y_{k}^{(n)^{\prime}}\right| \rightarrow \infty$ whenever $\gamma \neq \gamma^{\prime}$. Hence there are $m$ distinct terms of the form $w^{(n)}(\cdot+\gamma, \cdot), \gamma \in G$, in the expansion (2.6). Therefore (2.6) takes the form

$$
u_{k}-w^{(1)}-\sum_{n>1, \gamma \in G} w^{(n)}\left(\cdot+\gamma y_{k}^{(n)}, \cdot\right) \rightarrow 0 .
$$

It is easy to see that

$$
\left\|w^{(1)}\right\|^{2}+m \sum_{n>1}\left\|w^{(n)}\right\|^{2} \leq 1
$$

and

$$
\begin{aligned}
\int_{\mathbb{R}^{N-1}} b\left(x^{\prime}\right)\left|w^{(1)}\left(x^{\prime}, 0\right)\right|^{p} d x^{\prime} & +m \sum_{n>1} \int_{\mathbb{R}^{N-1}} b_{\infty}\left|w^{(n)}\left(x^{\prime}, 0\right)\right|^{p} d x^{\prime} \\
& =\lim _{k \rightarrow \infty} \int_{\mathbb{R}^{N-1}} b\left(x^{\prime}\right)\left|u\left(x^{\prime}, 0\right)\right|^{p} d x^{\prime}=c_{b, G} .
\end{aligned}
$$

Let $t_{1}=\left\|w^{(1)}\right\|^{2}$ and $t_{n}=m\left\|w^{(n)}\right\|^{2}$ for $n>1$. Then the relation (3.9) takes the form

$$
\sum_{n \geq 1} t_{n} \leq 1
$$

On the other hand, using (3.6), the definitions of the quantities $c_{b, G}, c_{\infty}$ and $c_{\infty, G}$, as well as (3.5) we derive the following inequality:

$$
c_{b, G} \leq c_{b, G} t_{1}^{p / 2}+m c_{\infty, G} \sum_{n>1} t_{n}^{p / 2} m^{-p / 2} \leq c_{b, G} t_{1}^{p / 2}+c_{b, G} \sum_{n>1} t_{n}^{p / 2} .
$$


This yields

$$
\sum_{n} t_{n}^{p / 2} \geq 1
$$

which combined with (3.11) implies that only one term $t_{n}$ is nonzero, say $t_{n_{\circ}}$. It follows from (3.9) and (3.10) that $n_{\circ}=1$.

4. Problem with the reverse penalty. In this section we prove Theorem 1.4. Let

$$
c_{b}:=\sup _{\|u\| \leq 1} \inf _{y^{\prime} \in \mathbb{Z}^{N-1}} \int b\left(x^{\prime}\right)\left|u\left(x^{\prime}-y^{\prime}, x_{N}\right)\right|^{p} d x .
$$

Let $u_{k}$ be a sequence satisfying, with some $y_{k}^{\prime} \in \mathbb{Z}^{N-1},\left\|u_{k}\right\| \leq 1$,

$$
\begin{aligned}
\int_{\mathbb{R}^{N-1}} b\left(x^{\prime}\right)\left|u_{k}\left(x^{\prime}+y^{\prime}, 0\right)\right|^{p} d x^{\prime} & \\
& \geq \int_{\mathbb{R}^{N-1}} b\left(x^{\prime}\right)\left|u_{k}\left(x^{\prime}+y_{k}^{\prime}, 0\right)\right|^{p} d x^{\prime} \rightarrow c_{b}, \quad y^{\prime} \in \mathbb{Z}^{N-1} .
\end{aligned}
$$

We will call any such sequence a maximizing sequence. Note that $\left|u_{k}\right|$ is then also a maximizing sequence, and in what follows we assume that $u_{k} \geq 0$. Moreover, $u_{k}\left(\cdot-y_{k}^{\prime}, \cdot\right)$ is also a maximizing sequence corresponding to $y_{k}^{\prime}=0$, so without loss of generality we set $y_{k}^{\prime}=0$. Let us apply Theorem 2.2, noting that since $u_{k} \geq 0$, all translated weak limits $w^{(n)}$ are non-negative.

Passing to the limit in (4.2) with $y^{\prime}=y_{k}^{(m)^{\prime}}+z^{\prime}, z^{\prime} \in \mathbb{Z}^{N-1}$, we obtain from Lemma 2.3,

$$
\begin{aligned}
& \int_{\mathbb{R}^{N-1}}\left(b\left(x^{\prime}\right)-b_{\infty}\right)\left|w^{(m)}\left(x^{\prime}+z^{\prime}, 0\right)\right|^{p} d x^{\prime} \\
& +\sum_{n} \int_{\mathbb{R}^{N-1}} b_{\infty}\left|w^{(n)}\left(x^{\prime}, 0\right)\right|^{p} d x^{\prime} \\
\geq & \int_{\mathbb{R}^{N-1}}\left(b\left(x^{\prime}\right)-b_{\infty}\right)\left|w^{(1)}\left(x^{\prime}, 0\right)\right|^{p} d x^{\prime}+\sum_{n} \int_{\mathbb{R}^{N-1}} b_{\infty}\left|w^{(n)}\left(x^{\prime}, 0\right)\right|^{p} d x^{\prime}=c_{b} .
\end{aligned}
$$

Note that $w^{(1)} \neq 0$, for if it were zero, (4.3) would imply that $w^{(m)}=0$ for every $m$, which yields $c_{b}=0$. This is a contradiction. Note also that (4.3) with $m=1$ implies

$$
\begin{aligned}
\int_{\mathbb{R}^{N-1}} b\left(x^{\prime}\right) \mid w^{(1)} & \left.\left(x^{\prime}, 0\right)\right|^{p} d x^{\prime} \\
& \left.\leq \int_{\mathbb{R}^{N-1}} b\left(x^{\prime}\right) \mid w^{(1)}\left(x^{\prime}+y^{\prime}, 0\right)\right)\left.\right|^{p} d x^{\prime}, \quad y^{\prime} \in \mathbb{Z}^{N-1} .
\end{aligned}
$$

Let $Y \subset \mathbb{Z}^{N-1}$ be the set of $y^{\prime}$ for which equality holds in (4.4). Note 
that $Y$ is finite, since

$$
\begin{aligned}
\lim _{\left|y^{\prime}\right| \rightarrow \infty} \int_{\mathbb{R}^{N-1}} b\left(x^{\prime}\right)\left|w^{(1)}\left(x^{\prime}+y^{\prime}, 0\right)\right|^{p} & =\int_{\mathbb{R}^{N-1}} b_{\infty}\left|w^{(1)}\left(x^{\prime}, 0\right)\right|^{p} d x^{\prime} \\
& >\int_{\mathbb{R}^{N-1}} b\left(x^{\prime}\right)\left|w^{(1)}\left(x^{\prime}, 0\right)\right|^{p} d x^{\prime} .
\end{aligned}
$$

Let $g_{y^{\prime}}(u)=\int b\left(x^{\prime}-y^{\prime}\right)\left|u\left(x^{\prime}, 0\right)\right|^{p} d x^{\prime} \in C^{1}\left(H^{1}\left(\mathbb{R}_{+}^{N}\right)\right)$. Assume that the function $w^{(1)} \in H^{1}\left(\mathbb{R}_{+}^{N}\right)$ does not belong to the positive cone generated by $g_{y^{\prime}}^{\prime}\left(w^{(1)}\right), y^{\prime} \in Y$. Then there exists a function $\left.v \in C_{0}^{\infty}\left(\mathbb{R}^{N}\right)\right|_{\mathbb{R}_{+}^{N}}$ with $\|v\|=1$ and an $\varepsilon>0$ such that $\left(w^{(1)}, v\right)<-2 \varepsilon$ and $\left(g_{y^{\prime}}^{\prime}\left(w^{(1)}\right), v\right)>2 \varepsilon$. Consider now a sequence $u_{k}+t v, t>0$. Then $\left\|u_{k}+t v\right\|^{2} \leq\left\|u_{k}\right\|^{2}+t^{2}+2 t\left(u_{k}, v\right) \leq$ $1+t^{2}-4 \varepsilon t \leq 1$ if $t \leq 4 \varepsilon$ and for all $t$ sufficiently small the functional $g_{y^{\prime}}\left(u_{k}+t v\right)$ satisfies

$$
\begin{aligned}
g_{y^{\prime}}\left(u_{k}\right. & +t v)=\int b\left(x^{\prime}-y^{\prime}\right)\left|\left(u_{k}+t v\right)\left(x^{\prime}, 0\right)\right|^{p} d x^{\prime} \\
& =\int b\left(x^{\prime}-y^{\prime}\right)\left|\left(w^{(1)}+t v\right)\left(x^{\prime}, 0\right)\right|^{p} d x^{\prime}+\sum_{n \geq 2} \int b_{\infty}\left|w^{(n)}\left(x^{\prime}, 0\right)\right|^{p} d x^{\prime}+o(1) \\
& \geq \sum_{n \geq 2} \int b_{\infty}\left|w^{(n)}\left(x^{\prime}, 0\right)\right|^{p} d x^{\prime}+\int b\left(x^{\prime}-y^{\prime}\right)\left|w^{(1)}\left(x^{\prime}, 0\right)\right|^{p} d x^{\prime}+\varepsilon t+o(1) \\
& =c_{b}+\varepsilon t+o(1) .
\end{aligned}
$$

Hence there is a $t_{0}>0$ and a $k(t)$ such that for every $k \geq k(t)$ and $0<t<t_{0}$

$$
g_{y^{\prime}}\left(u_{k}+t v\right) \geq c_{b}+\frac{1}{2} \varepsilon t
$$

Suppose that $y^{\prime} \notin Y$. Let

$$
\delta:=\inf _{y \in \mathbb{Z}^{N-1} \backslash Y} \int b\left(x^{\prime}-y^{\prime}\right)\left|w^{(1)}\left(x^{\prime}, 0\right)\right|^{p} d x^{\prime}-\int b\left(x^{\prime}\right)\left|w^{(1)}\left(x^{\prime}, 0\right)\right|^{p} d x^{\prime}
$$

In view of $(4.4), \delta \geq 0$. Since

$$
\begin{aligned}
\lim _{\left|y^{\prime}\right| \rightarrow \infty} \int b\left(x^{\prime}-y^{\prime}\right)\left|w^{(1)}\left(x^{\prime}, 0\right)\right|^{p} d x^{\prime} & =\int b_{\infty}\left|w^{(1)}\left(x^{\prime}, 0\right)\right|^{p} d x^{\prime} \\
& >\int b\left(x^{\prime}\right)\left|w^{(1)}\left(x^{\prime}, 0\right)\right|^{p} d x^{\prime},
\end{aligned}
$$

the mapping $y^{\prime} \mapsto \int b\left(x^{\prime}-y^{\prime}\right)\left|w^{(1)}\left(x^{\prime}, 0\right)\right|^{p} d x^{\prime}-\int b\left(x^{\prime}\right)\left|w^{(1)}\left(x^{\prime}, 0\right)\right|^{p} d x^{\prime}$ has a point of minimum over $y^{\prime} \in \mathbb{Z}^{N-1} \backslash Y$, and by definition of $Y$ the minimal value cannot be zero. 
Then

$$
\begin{aligned}
g_{y^{\prime}}\left(u_{k}\right. & +t v)=\int b\left(x^{\prime}-y^{\prime}\right)\left|\left(u_{k}+t v\right)\left(x^{\prime}, 0\right)\right|^{p} d x^{\prime} \\
& =\int b\left(x^{\prime}-y^{\prime}\right)\left|\left(w^{(1)}+t v\right)\left(x^{\prime}, 0\right)\right|^{p} d x^{\prime}+\sum_{n \geq 2} \int b_{\infty}\left|w^{(n)}\left(x^{\prime}, 0\right)\right|^{p} d x^{\prime}+o(1) \\
& \geq \sum_{n \geq 2} \int b_{\infty}\left|w^{(n)}\left(x^{\prime}, 0\right)\right|^{p} d x^{\prime}+\int b\left(x^{\prime}-y^{\prime}\right)\left|w^{(1)}\left(x^{\prime}, 0\right)\right|^{p} d x^{\prime}+C t+o(1) \\
& \geq c_{b}+\delta-C t+o(1) .
\end{aligned}
$$

Note that the $o(1)$ term is uniform in $t$ and $y$ (the latter due to Lemma 2.3), so that there is a $t>0$ such that for every $k$ sufficiently large, $g_{y^{\prime}}\left(u_{k}+t v\right)>$ $c_{b}+\frac{1}{2} \delta$ if $y^{\prime} \notin Y$. Combining this with a similar estimate for $y^{\prime} \in Y$, we deduce that for some $k$ and $t$, inf $y_{y^{\prime} \in \mathbb{Z}^{N-1}} g_{y^{\prime}}\left(u_{k}+t v\right)>c_{b}$. This is a contradiction. Thus $u$ is in the convex hull of $g_{y^{\prime}}^{\prime}$, which yields (1.3).

\section{References}

[1] H. Brézis and E. Lieb, A relation between pointwise convergence of functions, Proc. Amer. Math. Soc. 88 (1983), 486-490.

[2] M. Esteban and P.-L. Lions, A compactness lemma, Nonlinear Anal. 7 (1983), 381385.

[3] E. Lieb, Sharp constants in the Hardy-Littlewood-Sobolev and related inequalities, Ann. of Math. 118 (1983), 349-374.

[4] P.-L. Lions, The concentration-compactness principle in the calculus of variations. The limit case. I, Rev. Mat. Iberoamericana 1 (1985), 145-201.

[5] I. Schindler and K. Tintarev, An abstract version of the concentration compactness principle, Rev. Mat. Complut. 15 (2002), 417-436.

[6] X. P. Zhu and D. M. Cao, The concentration-compactness principle in nonlinear elliptic equations, Acta Math. Sci. 9 (1989), 307-327.

Jan Chabrowski

Department of Mathematics

University of Queensland

St. Lucia, 4072 Qld, Australia

E-mail: jhc@maths.uq.edu.au
Kyril Tintarev Department of Mathematics Uppsala University

SE-751 06 Uppsala, Sweden E-mail: kyril.tintarev@math.uu.se 